\title{
Lessons from the Effective Implementation of a Hybrid Virtual Clinical Model for Surgical Clerkships during the Covid Pandemic: Impact on Academic Performance, Burnout and Surgical Interest
}

\section{David Eugenio Hinojosa-Gonzalez}

Monterrey Institute of Technology and Higher Education

Andres Roblesgil-Medrano

Monterrey Institute of Technology and Higher Education

Victor Segura-lbarra

Monterrey Institute of Technology and Higher Education

Armando Tellez

Monterrey Institute of Technology and Higher Education

Jose Diaz-Elizondo

Monterrey Institute of Technology and Higher Education

Eduardo Flores-Villalba ( $\boldsymbol{\nabla}$ eduardofloresvillalba@tec.mx )

Monterrey Institute of Technology and Higher Education

\section{Research Article}

Keywords: Covid, Pandemic, Online Learning, Virtual Teaching, Academic Performance, Burnout

Posted Date: December 2nd, 2020

DOI: https://doi.org/10.21203/rs.3.rs-110618/v1

License: (c) (i) This work is licensed under a Creative Commons Attribution 4.0 International License.

Read Full License 


\section{Abstract}

Objective: Determine the academic and psychosocial impact of a hybrid-virtual surgical clerkship in medical students during the Covid Pandemic.

Background: The Covid-19 pandemic has forced universities to modify their academic curriculum to adapt and overcome never before seen adversities. Herein we present the implementation of a Hybrid Virtual Clinical Model for Surgical Clerkships and its comparison against traditional face-to-face learning.

Methods: We surveyed 47 medical students prior to their first-ever clerkship using Maslach's Burnout Inventory and a questionnaire regarding their interest in surgery as a career path before and after their clerkship. Burnout rates, clinical exposure, academic performance, and surgical interest were analyzed and compared against results from prior year students on a traditional program.

Results: Baseline burnout was significantly lower in the hybrid model $(15.4 \%$ vs. $2.6 \%, p=0.048)$. The same was seen on alternate burnout rates $(33.3 \%$ vs. $10.3 \%, p=0.02)$, differences remained after three months of clerkship ( $23.1 \%$ vs. $5.1 \%, p=0.023)$. When comparing different domains such as professional fulfillment, depersonalization, and fatigue, the hybrid model did overall better. Clinical exposure hours were statistically significant between both groups (954.32 vs. $276.33, p=0.001$ ), however, when considering virtual clinical hours, no significant differences were found. Despite the hybrid group having a lower midterm exam score (68 vs. $77.49, p=0.001)$, final exam scores were similar between groups (74.97 vs. $75.89, p=0.662)$.

Conclusion: We believe our model may be useful for other programs to maximize clinical exposure while minimizing the risk of exposure, maintaining maximum academic performance, and preserving the surgical interest of rotating alumni.

ACGME Competencies: Practice-Based Learning and Improvement

\section{Introduction}

The Covid-19 pandemic has forced humanity to rethink every aspect of their life that requires physical human-to-human interaction. This has led to extensive measures, ranging from enforcement of mandatory face masks, social distancing and the extensive use of online platforms in many areas. The latter greatly impacted the education realm, allowing schools to continue with their current curriculum in adapted or hybrid online environments. Traditional medical education and training requires on-site learning, demanding physical presence and interaction for an adequate clinical formation, however, what happens when the learning environments are the wolves' den where students are most exposed to the virus during a global pandemic? Online learning environments are the only setting in which safety can be guaranteed for students. This has left medical students in a limbo. Medical schools around the world have faced similar challenges, not only from the academic institution perspective but also from the student's point of view ${ }^{1,2}$. Should they be considered physicians and actively deployed as such, as some 
countries have when they are advanced in their training?, or should they still be considered students and be kept safe at home? ${ }^{3-6}$

Clinical environments are not only a powerful learning tool but also provide students with opportunities to participate in patient care and learn to perform clinical histories, physical examinations among other needed skills, thus active participation in supervised and relatively controlled environments is required to master the art of being a physician. 7,8

In their article, "I'm getting ready to help: response from medical schools to Covid19" by Valdez, et al., dean at our institution, the School of Medicine and Health Sciences of TecSalud, clearly highlights that the degree of responsibility and decision-making that students undertake is directly related to their stage of medical formation and differs within medical disciplines. ${ }^{9}$ Since the medical education of students must continue, a common challenge that required an immediate solution for medical schools worldwide is "how to best accommodate students safely while balancing their education?". ${ }^{10}$ This sets the basis towards the creation of a learning hybrid class model. ${ }^{9}$

We believe that the proposed virtual hybrid program can offset to an important degree the loss of clinical exposure, promote critical thinking and retain or increase interest in surgery, thus aiding clerkships around the world in the management of student formation during the pandemic. ${ }^{11}$

\section{Methodology}

This study was performed with prior consideration from the Internal Ethics Review Board as well as Science committee, and was performed in accordance to institutional regulations which are in accordance to national regulations established in the "Ley Mexicana General de Salud en Materia de Investigación, Artículo 17" (Mexican General Health Law) and abides by the norms established in the Declaration of Helsinki of 1964 as well as its revision in 2012. All surveyed participants agreed to the recollection of their data and signed an informed consent. Within the explanation of the study, it was clearly expressed that their participation or decline would have no effect on their grades or treat from the researchers.

The term "burnout" was first introduced in $1974 .{ }^{12}$ Maslach later defined burnout as a psychological syndrome involving emotional exhaustion, depersonalization, and a diminished sense of personal accomplishment that occurred among various professionals who work with other people in challenging situations. ${ }^{13}$ The Maslach Burnout Inventory (MBI) is the most commonly used instrument for measuring burnout. ${ }^{14}$ The MBI captures three dimensions of burnout: emotional exhaustion (EE), depersonalization (DP), and personal accomplishment (PA). The MBI has demonstrated in literature to be an excellent research tool to evaluate Burnout. ${ }^{15,16}$

The undergraduate surgical clerkship department constantly collects consenting student's data for continuous academic improvement and student wellbeing. This is completely optional and students who 
decline to participate face no consequence or point deduction. Baseline Maslach Scores are obtained at the start of every rotation, and surveys are reapplied every 6 weeks. Gathered student data include age, gender, alcohol and tobacco consumption, whether they are local, out of state or international students, average amount dedicated to studying, hospital and leisure activities and cohabitants. The order of their rotations is also registered. Clinical data gathered includes the amount and type of patients seen in the outpatient clinic, as well as how many surgeries they participated in, type of surgery and various other clinical activities. Academic data includes midterm and final exam scores as well as their performance in weekly tests, class attendance, workshop attendance and case simulation scores. Midterm and Final exams are heavily clinically focused and seek to assess student's diagnostic and therapeutic capabilities. These assessments tend to be different for every rotation by modifying variables such as patient data, grammar, shuffling answer options and rephrasing each question. Learning objectives, difficulty allocation and topic distribution have been conserved through exams. Difficulty is measured by historic percentage of wrong and right answers, Blackboard's own differential index and by our own metric on the amount of knowledge integration steps required to answer each question. This data has been gathered for over 5 years, totaling a data pool of over 500 students.

Prior to their first ever clinical rotation, consenting fifth-year students (MS3 US Equivalent) assigned to the surgical clerkship were surveyed prior to their beginning using Maslach's Burnout Inventory (MBI) amongst other data items. These students form Group $1(n=47)$. Following a baseline evaluation at the beginning of the study, Group 1 was surveyed again at 1 and 3 months follow-up. Additionally to the MBI, students were asked to answer a simple survey regarding their interest in surgery as a career path at the beginning and after their rotation ended. Pooled data from Group 1 was compared to pooled data from historical, previous fifth-year medical students (traditional face-to-face learning) during their first clinical rotation assigned to the surgical clerkship whose rotations were not affected by the current Covid-19 pandemic (Group 2 (historical control)) made up by 42 students, in an attempt to compare burnout rates as well as academic performance, as seen in figure 1 .

Group 1 was the first to participate in our institution's hybrid teaching model, in which clinical activities were reinstated partially in order to abide local sanitary regulations and to protect students from unnecessary contagion risk. The latter done under the premise of achieving maximum clinical training exposure. Group 1 attended two institutions, one public and one private as part of their normal training in university hospitals, both designated as Covid free, thus minimizing the risk of exposure.

Students on Group 1 were assigned on a Q4 on-call schedule. Those on-call attended the hospital, while those off-call students participated in online activities. During their $\sim 12$-hour calls, students were able to participate in surgeries of patients with negative SARS-CoV-2 PCR results. They were also involved in Emergency Department patient evaluation as well as preoperative and postoperative care when deemed safe. Students off-call participated in various online lectures, cases and grand rounds. Group 1 students, whether physically or via telecom attended grand rounds from 7:00 to 8:30 hrs., afterwards, attended fellowship lectures, journal clubs and academic lectures from 11:00 to 13:00 hrs., followed by interactive online surgical cases with expert physicians from 13:00 to 15:00 hrs. with alternating simulated handoffs 
every other day, all in which the main protagonist was the student. These sessions were followed by dynamic and interactive activities (i.e. surgical imaging evaluation, identifying key images and performing radiological dictation) watching surgical videos, identifying critical procedural steps as well as anatomical landmarks and performing postoperative notes. In total, Group 1 totaled $\sim 7$ hours of online "clinical" experience per day, while on-call students achieved 12 hours of clinical exposure per day. On average, Group 1 students achieved $\sim 50$ hybrid hours per week. Surveys were reapplied after one and three months of their surgical rotations.

Group 2 (historical control), involved daily clinical attendance and a Q3 on-call schedule, in which students spent over $\sim 70$ hours per week in a clinical setting, and per requisite to obtain a passing grade, participated in over 30 surgical procedures. These lower limit requirements were dropped for Group 1 by both a decrease in elective surgical procedures and to safeguard students.

For Group 2 (historical control), classes were held each Thursday at the same classroom from 08:00 to 14:00 hrs., with two 1-hour breaks scheduled by the attending professor's convenience. For Group 1, classes were held remotely every Thursday for the same time length through online platforms. Clinical performance evaluations were modified, with minimum procedure attendance cut out entirely. Academic curriculum and requirements were unaltered for the entirety of the study.

Academic performance was assessed with a midterm exam amid the 7th week and a final exam on the 12th week. The exam consisted of a 0 to 100 scale with a passing score of 70 and number of questions, complexity level (as reported by the testing platform's question-answer analysis) and subject allotment were not changed between groups. Exam's questions had equal distribution of content, level of difficulty and were based primarily on clinical cases. Only modifications in clinical scenario variables such as patients' gender, age, time of presentation as well as adding or subtracting non relevant details to avoid plagiarism were done. Students were offered a review session one week prior to the midterm exam, which was given by the same teacher utilizing the same slides. Our academic plan for the 12th week rotation and a sample weekly schedule are displayed in supplementary tables $1 \& 2$.

Statistical analysis was performed using SPSS v25 (IBM Corp, NY, US). Normality testing was performed through Kolmogorov-Smirnov. T-Student and ANOVA were used for parametric and Mann-Whitney-U and Kruskall Wallis for nonparametric testing. Chi-Square and Fisher's exact test were employed to compare frequencies.

\section{Results}

\section{Burnout Rates}

\section{Baseline}

Baseline burnout measurements of students prior to their first clinical rotation were significantly different, with students in Group 2 (historical control) presenting a 15.4\% (6) prevalence of burnout compared to 
2.6\% (1) of students in Group $1(\mathrm{p}=0.048)$. These differences were also true for alternate burnout rates, with Group 2 (historical control) totaling a 33.3\% (13) of alternate burnout compared to $10.3 \%$ in Group 1 (4) $(p=0.02)$.

\section{Follow up}

After three months of clinical rotations, in both Group 1 and Group 2 (historical control) differences in burnout rates remained, with Group 2 (historical control) displaying a $23.1 \%$ (9) of burnout rate compared to $5.1 \%$ (2) in Group 1 ( $p=0.023$ ). These represented a $7.7 \%$ and $2.5 \%$ increase in burnout during the first month, with a statistically significant difference $(p=0.003)$. This was also true for alternate burnout rate, with Group 2 (historical control) showing a $56.4 \%$ burnout rate compared to $7.7 \%(p=0.001)$ in Group 1.

\section{Changes in Burnout Scores}

Fifth-year medical students during their first clinical rotation in Group 2 (historical control) experienced significant alterations in their scores in various domains, such as decrease in professional fulfillment from 36.35 (24.16 - 38.61) to $32.17(28.65$ - 35.71) $(p=0.001)$, as well as increases in depersonalization from $11.38(9.03-13.73)$ to $14.38(11.98-16.79)(p=0.001)$ and fatigue from $29.15(25.78-32.53)$ to 36.17 (32.47 - 39.89) ( $p=0.001)$. Students in Group 1 had overall better baseline scores, and showed a significant decrease in fatigue $23.00(20.15-25.85)$ to $22.92(20.24-25.61)(p=0.001)$, slight decrease in personal fulfilment from $40.41(39.13-41.69)$ to $38.76(36.88-40.66)(p=0.03)$ and non-significant changes in depersonalization $5.07(3.74-6.42)$ to $5.39(4.14-6.58)(p=0.14)$.

\section{Clinical Exposure}

Thirty-seven students from Group 2 (historical control) achieved a mean $954.32( \pm 95.58)$ clinical exposure hours compared to Group 1, which achieved a mean $276.33( \pm 31)$ hours of clinical exposure $(p=0.001)$. However, when also considering virtual clinical hybrid hours invested in case presentations and grand round sessions by students, no significant differences were found between total hours exposed, with Group 2 (historical control) achieving $954.32( \pm 95.58)$ hours compared to $923.33( \pm 31.21)$ in Group 1 ( $p=0.001)$. Pearson correlation of physical clinical hours achieved during the first 6 weeks and midterm exam showed a significant correlation index of $0.421(p=0.001)$.

\section{Academic Performance}

Decreased clinical exposure might have reflected in the significant differences in midterm evaluation grades, with Group 1 achieving a mean score of $68( \pm 9.40)$ compared to $77.49( \pm 9.04)$ in Group 2 (historical control) ( $\mathrm{p}=0.001)$. These translated into an $84.2 \%$ (32) pass rate in Group 2 (historical control) to a $42.6 \%(20)$ of Group $1(p=0.001)$. Final score grades, however, were similar between groups, with Group 2 (historical control) scoring a mean of $74.97( \pm 6.97)$ versus $75.89( \pm 10.84)$ in Group $1(p=0.662)$. This interesting finding might be related to a longer period of adaptation for Group 1 to clinical scenarios which are commonly tested in exams and a delay in accumulation of clinical hours. These translated into 
an $86.5 \%$ pass rate for Group 2 (historical control) compared to an $82.1 \%$ pass rate for Group 1 $(p=0.596)$.

\section{Surgical Interest}

When surveyed, 19 Group 2 (historical control) students who answered our survey expressed their interest or disinterest for surgery at the beginning and end of the rotation. Of these, $10(52.2 \%)$ expressed disinterest, two $(10.5 \%)$ expressed ambivalence or neutrality and seven $(36.8 \%)$ expressed to be interested or highly interested in surgery. This contrasts to students in Group 1 in which a total of 30 students answered the survey at both the starting and ending of the clinical clerkship. Ten (50.0\%) students expressed disinterest, eight (26.7\%) expressed neutrality and 12 (40.0\%) expressed interest in surgery. Positive changes in interest (recruitment), retention of interest (retention) and loss of interest (attrition) were identified in 10 and 23 students from Group 2 (historical control) and Group 1 respectively. Of these, in Group 2 (historical control) interest was lost in four $(40.0 \%)$ students, two $(20.0 \%)$ were recruited and four (40.0\%) were retained. In Group 1, three students (13.0\%) lost interest, 11 (47.8\%) were positively impacted by the rotation and shifted from disinterest or neutral to interest in surgery and nine $(39.1 \%)$ were retained. These changes however were not statistically significant $(p=0.181)$. Differences between exam grades and clinical hour exposure were not significant when grouping students by interest in surgery. All the previously mentioned results are summarized in table 1.

Table 1. Main findings and differences among study groups regarding burnout, academic performance, clinical exposure, and surgical interest. $p$ values $<0.05$ were considered as statistically significant. 


\begin{tabular}{|c|c|c|c|c|c|}
\hline & \multicolumn{2}{|c|}{ Hybrid online program, Group 1} & \multicolumn{2}{|c|}{ Face-to-face program, Group 2} & $p$ \\
\hline & \multicolumn{5}{|l|}{ Variable } \\
\hline Variable & \multicolumn{5}{|c|}{ Burnout rates } \\
\hline Baseline & \multicolumn{2}{|l|}{$2.6 \%(1)$} & \multicolumn{2}{|l|}{$15.4 \%(6)$} & 0.048 \\
\hline \multirow[t]{3}{*}{3 Months } & $5.1 \%(2)$ & & $23.1 \%(9)$ & & 0.023 \\
\hline & \multicolumn{5}{|c|}{ Changes in burnout scores } \\
\hline & Beginning & End & Beginning & End & \\
\hline $\begin{array}{l}\text { Professional } \\
\text { fulfillment }\end{array}$ & $\begin{array}{l}23.00 \\
(20.15 \text { to } \\
25.85)\end{array}$ & $\begin{array}{l}22.92 \\
(20.24- \\
25.61)\end{array}$ & $\begin{array}{l}36.35 \\
(24.16- \\
38.61)\end{array}$ & $p=0.001$ & \\
\hline Depersonalization & $\begin{array}{l}0.41 \\
(39.13- \\
41.69)\end{array}$ & $\begin{array}{l}38.76 \\
(36.88- \\
40.66)\end{array}$ & $\begin{array}{l}11.38 \\
(9.03- \\
13.73)\end{array}$ & $p=0.001$ & \\
\hline \multirow[t]{2}{*}{ Fatigue } & $\begin{array}{l}5.07(3.74 \\
-6.42)\end{array}$ & $\begin{array}{l}5.39 \\
(4.14- \\
6.58)\end{array}$ & $\begin{array}{l}29.15 \\
(25.78- \\
32.53)\end{array}$ & $p=0.001$ & \\
\hline & \multicolumn{4}{|c|}{ Academic grades (0-100 scale) } & \\
\hline Midterm exam & \multicolumn{2}{|l|}{$68 \pm 9.40$} & \multicolumn{2}{|l|}{$77.49 \pm 9.0$} & 0.001 \\
\hline \multirow[t]{2}{*}{ Final exam } & \multicolumn{2}{|c|}{$75.89 \pm 10.84$} & \multicolumn{2}{|l|}{$74.97 \pm 6.97$} & 0.662 \\
\hline & \multicolumn{4}{|c|}{ Clinical and virtual exposure } & \\
\hline $\begin{array}{l}\text { Real clinical } \\
\text { exposition (hours) }\end{array}$ & \multicolumn{2}{|l|}{$276.33 \pm 31$} & \multicolumn{2}{|l|}{$954 \pm 95.58$} & 0.001 \\
\hline \multirow{2}{*}{$\begin{array}{l}\text { Real clinical + } \\
\text { virtual exposition } \\
\text { (hours) }\end{array}$} & \multicolumn{2}{|c|}{$923.33 \pm 31.21$} & $954.32 \pm 95.5$ & & 0.001 \\
\hline & Preferences & & & & \\
\hline Recruit & $47.8 \%(11)$ & & $20 \%(2)$ & & 0.181 \\
\hline Retain & $39.1 \%(9)$ & & $40 \%(4)$ & & \\
\hline Attrition & $13 \%(3)$ & & $40 \%(4)$ & & \\
\hline
\end{tabular}

\section{Discussion}

The Covid-19 pandemic has required us to address questions about medical training, including both students and trainee's involvement in the care of infectious patients. Just as when evaluating the risks 
and benefits of any medication or surgical procedure on any given patient, the high probability that medical students in the hospital would become infected by SARS-CoV-2 and the need to reduce the expenditure of personal protective equipment (PPE) appeared to outweigh the educational benefits of student's involvement in everyday hospital activities, which obligated medical educational programs to restructure their teaching process. ${ }^{17}$

However, as the pandemic progressed, our institution deemed possible to return students to controlled clinical environments in which their safety could be maximized. Our results comparing clinical rotations differences in burnout rates remained, regardless of the teaching process. Data analysis in the pre-Covid group showed a significant difference against the hybrid learning group on variables such as decrease in professional fulfillment as well as increases in depersonalization and fatigue, demonstrating that the new hybrid learning method is linked to less professional burnout among medical students, however, its true extent is yet to be determined due to possible stress caused by the pandemic.

Prior studies have identified exposure to Covid-19 as a source of stress in trainees. These differences could be due to changes in schedules, activities or fears of contracting or spreading the disease. ${ }^{18} \mathrm{We}$ found the opposite in our students, however this difference is probably related to the degree and extents of shielding we were able to provide our students, nurturing a sense of safety and security in which their health was primordial. We believe decreased burnout rates in our cohort were also related to increased time for reading and studying, as exam anxiety has been identified as a trigger for stress, shifting the Yerkes-Dodson curve towards a more-optimal point. ${ }^{19}$ We had previously identified decreased burnout rates in our cohort of students when decreasing call frequency, as students had more time to rest and study. ${ }^{9}$ We believe that a constant fixed schedule also aided in reducing stress, as it was easier for students to know what they were supposed to be doing at each particular moment.

An observational study made in the Taipei Medical University in which 109 medical students participated in an early clinical exposure vs. non-early clinical exposure program revealed that a student's learning environment had a significant positive predictive power on standardized test scores, which could translate to the studied group in this study, since our hybrid model group had never before had any type of clinical exposure. ${ }^{19}$ This could explain the initial underperformance in exam's, taking longer to assimilate and develop critical clinical thought process that is usually aided in development by attendings and residents during in hospital activities.

A retrospective cohort study by Cortez, et al. at the University of Cincinnati evaluated the impact of medical student interest in surgery on clerkship performance and career choice on 62 third-year medical students. The study revealed that although there was no difference in overall burnout rate, students interested in surgery had a lower emotional exhaustion score at the completion of the rotation $(19.5 \% \mathrm{vs}$ $24.5 \%, p=0.03$ ) compared with those with no interest in a surgical career. Regardless of reported surgery career interest, there was no difference between students with no interest compared to students with interest with regard to final clerkship numeric grade ( $85.3 \%$ vs. $86.3 \%, p=>0.05) .{ }^{20}$ This matches our findings in academic performance being unrelated to interest in surgery. In our cohort, interest was 
retained even when less exposed to hands-on clinical experience, however, further studies are needed to clarify this phenomenon, as students were constantly immersed in surgical cases either through online case presentations, grand rounds, and classes by subspecialist attendings that included videos and interactive activities. Activities included students watching surgical procedures on video, identifying key images and critical steps or anatomical landmarks, and doing a mock post-surgical note. This type of activities, while not the same as actual hands-on surgical assistance, might allow students to develop a sense of autonomy and creativity envisioning themselves as surgeons. These activities might help retain and grow surgical interest.

When comparing online versus offline learning in undergraduate medical education, a systematic review and meta-analysis published by Pei et al., affirmed a statistically significant difference between online (70.1 $\pm 3 \%)$ and offline learning $(55.8 \pm 3 \%)$ for retention test scores, being online learning more effective than offline learning (SMD $=4.64 ; 95 \% \mathrm{Cl}: 3.19,6.09 ; \mathrm{p}<0.00001)$. The same applied to post-test scores, in which the online learning group had statistically significant $(Z=4.17 ; p<0.0001)$ higher post-test scores compared to the offline learning group $(S M D=0.81 ; 95 \% \mathrm{Cl}: 0.43,1.20) .{ }^{21}$

Surprisingly, despite the relative increase in the number of stay-at-home hours and less on-call and postcall hours, Group 1 scored a lower mean on the midterm exam than Group 2 (historical control). This could be caused by a lower number of hours of clinical exposure. However, this was later offset by their performance in the final exam. We believe there is a steep learning curve to clinical exposure needed to approach clinical scenarios that is easier to overcome when constantly immersed in clinical scenarios.

This meta-analysis showed that there is no evidence that offline learning works better, and that online learning has advantages to enhance knowledge and skills when compared to offline learning. When it was evident that the COVID-19 pandemic was going to be longer than initially anticipated, academic life needed to adapt. Our university applies all available resources to facilitate continuing education for our medical students. COVID-19 challenged and tested our capabilities. The silver lining of our response to this pandemic is that when technological resources are available, online and remote learning do not impact medical training. In fact, this opens new horizons and suggests new academic opportunities, for example; classes with a larger number of students not limited by classroom capacity. Capability of universities to provide medical education in different geographical locations within the city, country or even continent. Capabilities to access information on-demand, that could allow proper and detailed review of information and a larger academic schedule flexibility. This online surgical program could also prepare medical students to a new surgical world we are currently living in which robotics and informatics is commonly part of a surgical procedure. Of course, this also brings new challenges. This would force all students to have proper internet access at all times which in some cases can be difficult and not always possible. However, these initiatives could allow academic institutions to free resources that could be focused on enhancing their remote online training to facilitate access to students.

Our study faces various limitations, firstly, clinical evaluation per se is absent, and a clinical focused academic evaluation is used as a surrogate for actual clinical performance. However, our academic 
evaluations are heavily clinically focused, assessing students' knowledge and capacity to identify the presentation of various diseases, as well as basic radiologic interpretation and surgical anatomy, with certain overlap on level of knowledge with that of junior residents. Another important limitation is the use of a non-standardized simple survey gauging surgical interest. Lastly, further limitations could arise from the control group being Q3 compared to the hybrid Q4, however, future studies could retrospectively compare more similar groups once the pandemic is over. Further studies could dive deeper into persistent virtual implementations and their impact on wellbeing in a context where they can be safely compared to face-to-face models.

\section{Conclusion}

Despite the current pandemic and its associated limitations related to the formation of students in surgical settings, we believe our model may be useful for other programs to maximize clinical exposure while minimizing contagion risk and still achieving maximum academic performance and retaining surgical interest in alumni. We hope our experience can be of use and extended to other programs in their attempts to navigate their commitment to the development of qualified students under these unseen circumstances and the lessons learned from this experience continue to build on effective virtual alternatives and teaching methods in the medical realm.

\section{Declarations}

\section{Acknowledgements}

The authors would like to thank all the volunteers that decided to participate in the survey for this manuscript. We thank the Department of Clinical Sciences for allowing us to perform such survey.

\section{References}

1. Sandhu, P., \& de Wolf, M. (2020). The impact of COVID-19 on the undergraduate medical curriculum. Medical Education Online, 25(1), 1764740.

2. Compton, S., Sarraf-Yazdi, S., Rustandy, F., \& Kumar Radha Krishna, L. (2020). Medical students' preference for returning to the clinical setting during the COVID-19 pandemic. Medical Education.

3. Lapolla, P., \& Mingoli, A. (2020). COVID-19 changes medical education in Italy: will other countries follow?.

4. Early Graduation at NYU Grossman School of Medicine Sends New Doctors to Join COVID-19 Fight. (2020, April 05). https://nyulangone.org/news/early-graduation-nyu-grossman-school-medicinesends-new-doctors-join-covid-19-fight

5. Antman, K. (2020, March 26). Important Message to the Class of 2020. https://www.bumc.bu.edu/2020/03/26/3-26-important-message-to-the-class-of-2020/ 
6. Sharif, S. P. (2020). UK medical students graduating early to work in the COVID-19 pandemic. Psychological Medicine, 1-4.

7. Isba R, Boor K. Creating a learning environment. In: Dornan T, Mann K, Scherpbier A, Spencer J, editors. Medical Education Theory and Practice. Edinburgh: Churchill Livingstone Elsevier; 2011. pp. 99-114.

8. Boor K, Scheele F, van der Vleuten CP, Teunissen PW, den Breejen EM, Scherpbier AJ. How undergraduate clinical learning climates differ: a multi-method case study. Med Educ. 2008;42:1029-36.

9. Valdez V, López M, Jiménez M, Díaz Elizondo JA, Dávila Rivas JA, Olivares S. Me preparo para ayudar: respuesta de escuelas de medicina y ciencias de la salud ante COVID-19. Inv Ed Med. 2020;9:1-11. http://riem.facmed.unam. mx/sites/all/archivos/prensa/COVID-19_RIEM.pdf

10. Calhoun, K. E., Yale, L. A., Whipple, M. E., Allen, S. M., Wood, D. E., \& Tatum, R. P. (2020). The impact of COVID-19 on medical student surgical education: implementing extreme pandemic response measures in a widely distributed surgical clerkship experience. The American Journal of Surgery.

11. Hinojosa-Gonzalez, D. E., Farias, J. S., Tellez-Giron, V. C., Aguirre-Villarreal, D., Brenes-Castro, D., \& Flores-Villalba, E. (2020). Lower Frequency of Call Shifts Leads to Higher Attendance, Higher Academic Performance, and Less Burnout Syndrome in Surgical Clerkships. Journal of Surgical Education. doi:10.1016/j.jsurg.2020.07.043

12. Freudenberger, H. J. (1974). Staff burn-out. Journal of social issues, 30(1), 159-165.

13. Maslach, C. (2003). Burnout: The cost of caring. Ishk.

14. Maslach, C., Jackson, S. E., Leiter, M. P., Schaufeli, W. B., \& Schwab, R. L. (1986). Maslach burnout inventory (Vol. 21, pp. 3463-3464). Palo Alto, CA: Consulting psychologists press.

15. Greenglass, E. R., Burke, R. J., \& Fiksenbaum, L. (2001). Workload and burnout in nurses. Journal of community \& applied social psychology, 11(3), 211-215.

16. Hastings RP, Horne S, Mitchell G. Burnout in direct care staff in intellectual disability services: A factor analytic study of the Maslach Burnout Inventory. Journal of Intellectual Disability Research. 2004;48(3):268-273.

17. Jorge, E., Irma, E., José, A., Mary, A., Alejandro, T. Q., Ramón, I., \& Lydia, Z. G. (2020). The role of the medicine student in COVID-19 pandemic. A shared responsibility. Cirugía y cirujanos, 88(4), 399-401.

18. Kannampallil TG, Goss CW, Evanoff BA, Strickland JR, McAlister RP, Duncan J (2020) Exposure to COVID-19 patients increases physician trainee stress and burnout. PLoS ONE 15(8): e0237301. https://doi.org/10.1371/journal. Pone.0237301

19. Tang, K. P., Chen, C. Y., Wu, M. S., Chen, T. T., Wu, B. W., \& Tsai, P. F. (2019). Correlation between early clinical exposure environment, attitudes toward basic medicine, and medical students' basic science learning performance. BMC medical education, 19(1), 183.

20. Cortez, A. R., Winer, L. K., Kassam, A. F., Kuethe, J. W., Athota, K. P., \& Quillin III, R. C. (2019). The impact of medical student burnout on surgery clerkship performance. Journal of surgical education, 76(5), 1241-1247.

Page 12/14 
21. Pei, L., \& Wu, H. (2019). Does online learning work better than offline learning in undergraduate medical education? A systematic review and meta-analysis. Medical education online, 24(1), 1666538.

Figures

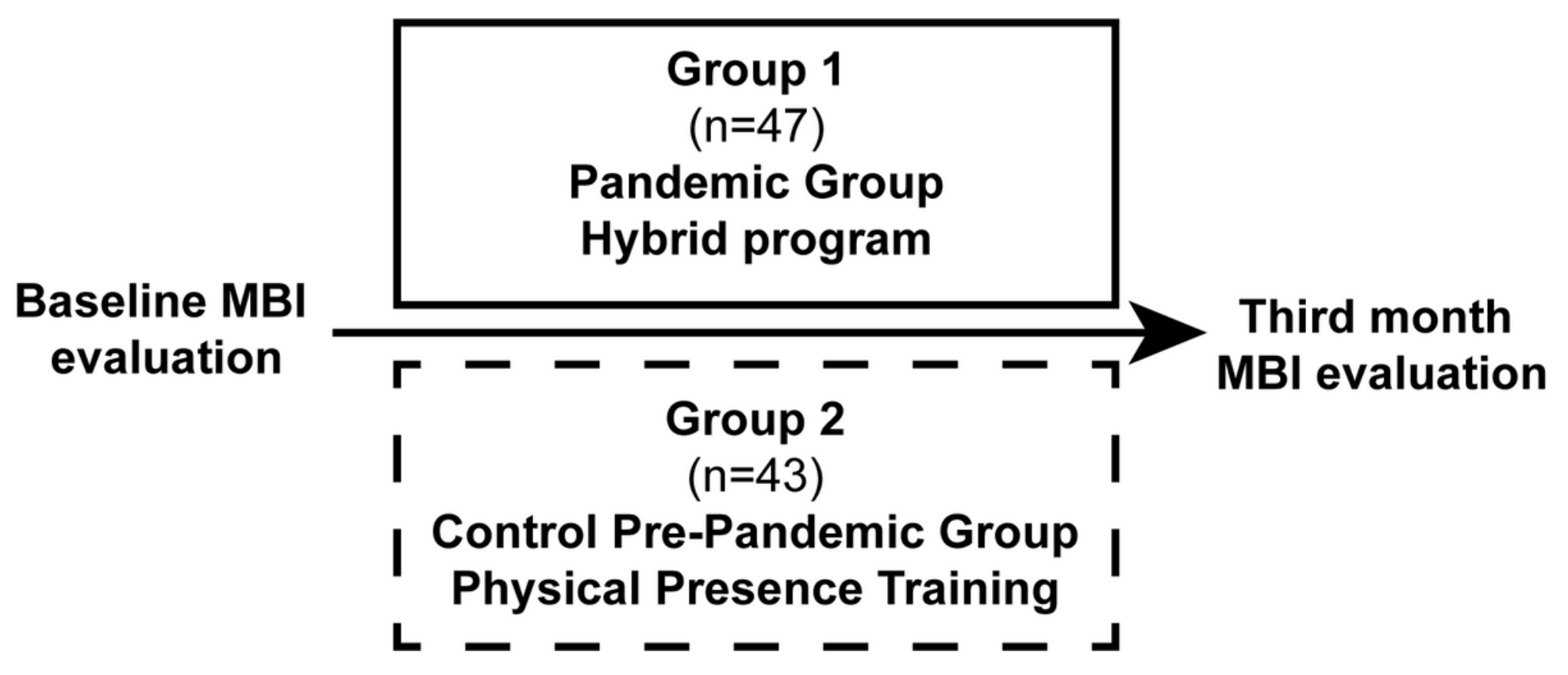

Figure 1

Diagram of study groups and timeline of MBI assessment

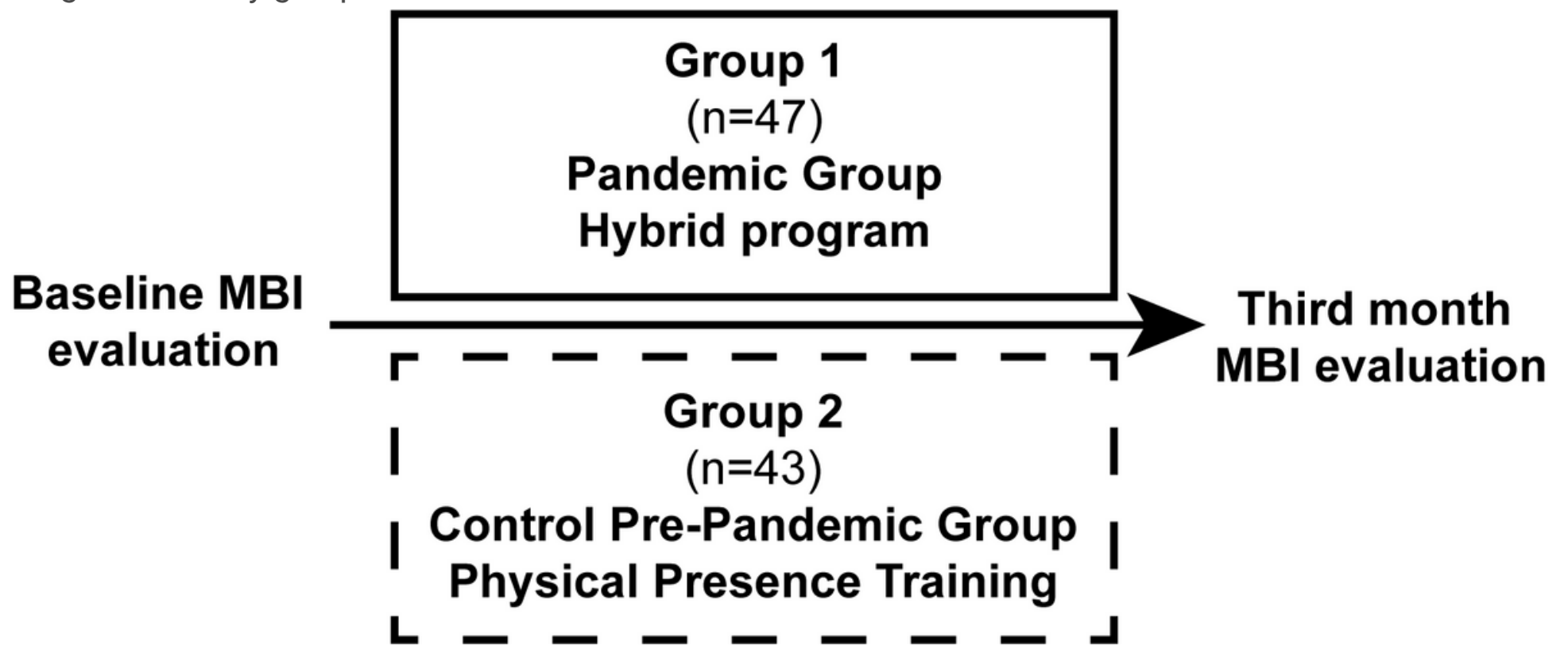

Figure 1 


\section{Supplementary Files}

This is a list of supplementary files associated with this preprint. Click to download.

- Supplemental.docx

- Supplemental.docx 